Dieses Dokument ist eine Zweitveröffentlichung (Postprint Version) / This is a self-archiving document (accepted version):

Christian Meerbach, Cong Wu, Steven C. Erwin, Zhiya Dang, Anatol Prudnikau, Vladimir Lesnyak

Halide-Assisted Synthesis of Cadmium Chalcogenide Nanoplatelets Erstveröffentlichung in / First published in:

Chemistry of Materials. 2019, 32 (1), S. 566-574. ACS Publications. ISSN: 1520-5002. DOI: https://doi.org/10.1021/acs.chemmater.9b04438

Diese Version ist verfügbar / This version is available on:

https://nbn-resolving.org/urn:nbn:de:bsz:14-qucosa2-743231 


\title{
Halide-Assisted Synthesis of Cadmium Chalcogenide Nanoplatelets
}

\author{
Christian Meerbach, ${ }^{1}$ Cong Wu, ${ }^{1}$ Steven C. Erwin, ${ }^{2}$ Zhiya Dang, ${ }^{3}$ Anatol Prudnikau, \\ Vladimir Lesnyak ${ }^{1 *}$
}

${ }^{1}$ Physical Chemistry, TU Dresden, Bergstr. 66b, 01062 Dresden, Germany

${ }^{2}$ Center for Computational Materials Science, Naval Research Laboratory, Washington DC 20375, USA

${ }^{3}$ Nanochemistry Department, Istituto Italiano di Tecnologia, Via Morego 30, 16163 Genova, Italy

\begin{abstract}
Atomically flat colloidal semiconductor CdSe nanoplatelets (NPLs) with precisely controlled thickness possess a range of unique optoelectronic properties. Here we study the growth of CdSe, CdTe, and CdS NPLs with the aim of synthesizing thicker NPLs in order to extend their optical activity further into the lower energy/larger wavelength range. We employ cadmium halides, which lead to faster reaction kinetics as confirmed by a control experiment employing cadmium hydroxide as a Cdprecursor. Addition of halides in all cases led to the formation of thicker NPL species, as compared with the corresponding syntheses without these additives. Analysis of a recent theoretical model of the platelet growth mechanism confirms an earlier suggestion that reducing both the reaction enthalpy and the surface energy of $\mathrm{CdSe}$, by replacing acetate ligands with chloride ions, should indeed lead to thicker NPLs as observed. We observed a formation of $\mathrm{Cd}^{0}$-metal nanoparticles in the first stage of the synthesis by preparing the Cd-precursor, which is another key finding of our work. We assume that these particles can serve as an active cadmium source facilitating the growth of the NPLs. The resulting 6 ML CdSe NPLs exhibited bright photoluminescence with quantum yield of up to $50 \%$, exceptionally narrow spectrum centered at $582 \mathrm{~nm}$ with full width at half maximum of approx. $11 \mathrm{~nm}$ and small Stokes shift of $2 \mathrm{~nm}$. Moreover, we demonstrated the synthesis of heterostructured core/crown and core/shell CdSe/CdS NPLs based on 6 ML CdSe platelets, which also exhibited bright fluorescence. This work shows the possibility to overcome energetic barrier limiting the size (thickness) control by using appropriate promoters of the growth of CdSe, CdTe and CdS 2D structures.
\end{abstract}

\section{INTRODUCTION}


Optoelectronic properties of semiconductor nanoplatelets (NPLs) are dictated by a strong quantum confinement only within their thickness, which can be precisely controlled by conditions of the colloidal synthesis, mainly by the reaction time and temperature. ${ }^{1-3}$ This unprecedented control has already yielded atomically flat NPLs made of a few monolayers of CdS, CdSe, and CdTe. ${ }^{4}$ Among them, CdSe NPLs exhibit the most striking properties demonstrating many advantages over corresponding quantum dots, such as exceptionally narrow photoluminescence (PL) spectra, short lifetimes, giant oscillator strength, low laser threshold, and high absorption cross section. ${ }^{4-9}$ These make NPLs a very promising material for application in light emitting diodes, lasers, field-effect transistors, photodetectors, etc. ${ }^{5,8-14}$ Recently, the formation of cadmium chalcogenide two-dimensional NPLs was rationalized by the Norris group, which developed a predictive, materials-specific theoretical model of the growth instability that leads to flat platelets. ${ }^{15,16}$ The authors explained the growth of anisotropic NPLs even from isotropic materials by considering the barrier for nucleating the growth of new material in two regimes: on wide facets and on narrow facets. Nucleation on the narrow facets is easier but, as their thickness increases, this nucleation barrier approaches the barrier for growth the wide facet. This naturally leads to a maximum value for the thickness, which was assumed in ref. ${ }^{15}$ to be 5 monolayers (MLs) because thicker platelets of CdSe had not yet been observed. However, this maximum value depends on several materials parameters and hence can, in principle, be deliberately altered by changing those parameters. In addition, other strategies outside the scope of the growth model have also been explored, for example growing additional CdSe MLs on preformed NPLs via the colloidal atomic layer deposition (c-ALD) method developed by Ithurria and Talapin. ${ }^{17}$ Using this method, Chu et al. recently synthesized CdSe NPLs with thicknesses up to 11 MLs. ${ }^{14}$ However, the authors did not demonstrate any PL properties of these thick NPLs, implying that c-ALD does not yield defect-free crystal structure available from the direct synthesis of the NPLs at elevated temperature.

During the preparation of this paper, Moreels and co-workers reported the first successful synthesis of CdSe NPLs with thickness exceeding 5 ML, i.e. 6, 7 and 8 ML platelets, via switching their growth from $2 \mathrm{D}$ to $3 \mathrm{D}$ regime in the presence of cadmium chloride. ${ }^{18}$ Despite this great achievement in the growth control of CdSe NPLs, their optical properties, in particular PL quantum yields (QYs) and fluorescence purity require a further improvement. Thus, for example 6 ML thick NPLs, a next member after the well-developed 5 ML thick platelets, reported in that work had PLQY of $14 \%$. Furthermore, as noted by the authors, all thicker NPLs exhibited increased heterogeneous size broadening, that is, they contained some amounts of 1 ML thinner and thicker NPLs, which eventually resulted in broadening of the PL bands. Later on, the Talapin group has reported a successful direct synthesis of 6 ML thick NPLs. ${ }^{19}$ In order to grow thicker NPLs, they modified recipe for the syntehsis of 5 ML CdSe platelets by 
introducing aqueous solution of cadmium chloride in addition to cadmium acetate. In their work, hovewer, the authors did not provide a full insight into the mechanism of the growth of the thicker species.

In this work, we systematically studied the impact on the growth of CdSe, CdS and CdTe NPLs of adding various cadmium halides $\left(\mathrm{CdF}_{2}, \mathrm{CdCl}_{2}, \mathrm{CdBr}_{2}\right.$, and $\left.\mathrm{CdI}_{2}\right)$ to the main Cd-precursor (cadmium acetate, or cadmium hydroxide in the control experiments). Our results reveal that these halides accelerate the NPLs nucleation and growth, most likely by altering the reacting surface of the platelets and thus changing its energy. Furthermore, we observed formation of metallic Cd nanoparticles resulting from the reaction between $\mathrm{CdO}$ and myristic acid in 1-octadecene (ODE); these $\mathrm{Cd}$ nanoparticles play the role of Cd reservoirs, similar to that reported for the synthesis of CdTe quantum dots ${ }^{20}$. Within the family of cadmium chalcogenides, CdSe NPLs demonstrate the most striking optical properties and hence we focused our investigation mainly on this class of particles. The proposed modification of the method allows for the growth of pure population of 6 ML thick CdSe NPLs. In addition, we also observed a clear sign of the formation of 7 ML NPLs in this direct synthesis. We also found that the addition of halides promoted the growth of NPLs in the lateral directions. The resulting NPLs exhibited high-colorpurity PL with narrow spectrum having full width at half maximum (FWHM) of approx. $11 \mathrm{~nm}$ (39 $\mathrm{meV}$ ), centered at $582 \mathrm{~nm}$, PLQY of up to $50 \%$, and a small Stokes shift of $2 \mathrm{~nm}$. Moreover, using these NPLs as cores, we synthesized heterostructured core/shell and core/crown CdSe/CdS NPLs which exhibited excellent optical properties. Although our main focus is CdSe NPLs, we also demonstrate that our findings are applicable to analogous $\mathrm{CdS}$ and CdTe 2D nanostructures, underlining the generality of our approach to thicker cadmium chalcogenide NPLs with optical properties extending to the lowerenergy regions of the visible spectrum.

\section{EXPERIMENTAL SECTION}

\section{Chemicals.}

Ammonium sulfide $\left(\left(\mathrm{NH}_{4}\right)_{2} \mathrm{~S}, 40-48 \%\right.$ in water), cadmium acetate $\left(\mathrm{Cd}(\mathrm{OAc})_{2}, 99.995 \%\right)$, cadmium chloride $\left(\mathrm{CdCl}_{2}, 99.99 \%\right)$, cadmium iodide $\left(\mathrm{CdI}_{2}, 99.999 \%\right)$, cadmium nitrate tetrahydrate $\left(\mathrm{Cd}\left(\mathrm{NO}_{3}\right)_{2} \cdot 4 \mathrm{H}_{2} \mathrm{O}\right)$, cadmium oxide $(\mathrm{CdO}, 99.99 \%)$, zinc acetate dihydrate $\left(\mathrm{Zn}(\mathrm{OAc})_{2} \cdot 2 \mathrm{H}_{2} \mathrm{O}, 99 \%\right), 1-$ octadecene (ODE, 90\%), myristic acid (98.5\%), oleic acid (OlAc, 90\%), oleylamine (O1Am, 70\%), selenium powder ( $\mathrm{Se}, 100$ mesh, 99.99\%), and valeric acid $\left(\mathrm{C}_{5} \mathrm{H}_{10} \mathrm{O}_{2},>99 \%\right)$ were purchased from Sigma-Aldrich. Cadmium bromide $\left(\mathrm{CdBr}_{2}, 99 \%\right)$, cadmium fluoride $\left(\mathrm{CdF}_{2}, 99 \%\right)$, and sulfur flakes (S, $99.999 \%)$ - from $\mathrm{ABCR}$, cadmium acetate dihydrate $\left(\mathrm{Cd}(\mathrm{OAc})_{2} \cdot 2 \mathrm{H}_{2} \mathrm{O}, 99.99 \%\right)$ and tellurium powder (Te, 99.999\%) - from Chempur, ethanol (anhydrous, >99\%) - from Merck, n-hexane (95\%) - from 
VWR, formamide (FA, 99.5\%) - from Acros Organics, tri- $n$-octylphosphine (TOP, 97\%) - from Strem Chemicals.

\section{Synthesis of 6 ML CdSe NPLs.}

First, $70 \mathrm{mg}$ of $\mathrm{CdO}(0.54 \mathrm{mmol})$ and $340 \mathrm{mg}$ of myristic acid $(1.4 \mathrm{mmol})$ in $28 \mathrm{~mL}$ of ODE were degassed at $110^{\circ} \mathrm{C}$ for $30 \mathrm{~min}$ in a $50-\mathrm{mL}$ three-neck flask and then heated up to $305^{\circ} \mathrm{C}$ for $20-30 \mathrm{~min}$ under argon. During this heating step, the initially clear colorless solution turned to a green-greyish turbid dispersion. Second, after the preparation of the cadmium precursor, the reaction mixture was cooled down to $90^{\circ} \mathrm{C}$ and degassed once again to remove water vapors. In the next step we added $24 \mathrm{mg}$ of Se $(0.3 \mathrm{mmol})$ suspended in $2 \mathrm{~mL}$ of ODE by ultra-sonication for $30 \mathrm{~min}$, and then degassed the system. To grow $6 \mathrm{ML}$ CdSe NPLs the reaction temperature was raised up to $280^{\circ} \mathrm{C}$. While the temperature of the reaction mixture was increasing, $138.3 \mathrm{mg}$ of $\mathrm{Cd}(\mathrm{OAc})_{2}(0.64 \mathrm{mmol})$ and $81.2 \mathrm{mg}$ of $\mathrm{CdF}_{2}(0.54 \mathrm{mmol})$ were quickly added at $210^{\circ} \mathrm{C}$. The NPLs grew at $280^{\circ} \mathrm{C}$ for $40 \mathrm{~min}$. Finally, the growth was quenched by rapid cooling with a water bath and the addition of $2 \mathrm{~mL}$ of OlAc at $200^{\circ} \mathrm{C}$. Further, the solution was divided into two parts and filled in $50 \mathrm{~mL}$ centrifugation tubes, where $20 \mathrm{~mL}$ of a hexane:ethanol (3:1) mixture were added to each portion with subsequent centrifugation at $4000 \mathrm{rpm}(\mathrm{rcf}=1972 \mathrm{~g}$ ) for $5 \mathrm{~min}$ to separate the NPLs from CdSe nanoparticles typically formed as a byproduct. The precipitate was dissolved in $8 \mathrm{~mL}$ of hexane. To separate 5 ML NPLs from 6 ML NPLs we added a centrifugation step of the solution at $12000 \mathrm{rpm}$ for 10 min yielding a supernatant containing solely 6 ML-thick NPLs.

\section{CdSe/CdS Core/Crown NPLs.}

Core/crown CdSe/CdS heterostructured NPLs were synthesized according to ref. ${ }^{21}$. Briefly, $500 \mu \mathrm{L}$ of 6 ML NPL stock solution (optical density of $15 \mu \mathrm{L}$ of this solution diluted with $1 \mathrm{~mL}$ hexane $=0.586$ at the heavy hole (hh)-absorption maximum) was mixed with $8 \mathrm{~mL}$ of ODE in a $25 \mathrm{~mL}$ three-necks flask and degassed at $100^{\circ} \mathrm{C}$ to remove hexane. We then injected a precursor solution for crown growth into this core solution. The CdS crown precursor was prepared as follows. First, $\mathrm{Cd}(\mathrm{OAc})_{2} \cdot 2 \mathrm{H}_{2} \mathrm{O}(160 \mathrm{mg}$, $0.6 \mathrm{mmol}$ ) and $130 \mu \mathrm{L}$ of OlAc were stirred in $1 \mathrm{~mL}$ of $\mathrm{ODE}$ at $210^{\circ} \mathrm{C}$ until a yellow-turbid mixture was formed. The mixture was ultrasonicated for a few minutes to facilitate dissolving of the salt. This solution was mixed with $1 \mathrm{~mL}$ of $0.1 \mathrm{M} \mathrm{S}-\mathrm{ODE}$ precursor solution (prepared via dissolving $25.6 \mathrm{mg}$ of sulfur $(0.8$ $\mathrm{mmol}$ ) in $8 \mathrm{~mL}$ of ODE in a glass vial at room temperature in a nitrogen filled glovebox overnight) at room temperature. The $0.1 \mathrm{M} \mathrm{S}-\mathrm{ODE}$ solution was prepared via dissolving $25.6 \mathrm{mg}$ of sulfur ( $0.8 \mathrm{mmol})$ in $8 \mathrm{~mL}$ of $\mathrm{ODE}$ in a glass vial at room temperature in the glovebox overnight. To prepare the heterostructured NPLs, $1.2 \mathrm{~mL}$ of the mixed solution was added continuously into 6 ML NPLs in ODE 
with $8 \mathrm{~mL} / \mathrm{h}$ rate at $230^{\circ} \mathrm{C}$ under argon flow. After the complete addition (within approx. 9 min), the reaction mixture was cooled down to room temperature and filled in 50-mL centrifugation tubes with 20 $\mathrm{mL}$ of a hexane:ethanol (3:1) mixture. After $10 \mathrm{~min}$ centrifugation at $5100 \mathrm{rpm}$, the precipitate was dissolved in $2 \mathrm{~mL}$ of hexane.

\section{CdSe/CdS Core/Shell NPLs.}

The growth of the CdS shell was performed following a recently published recipe. ${ }^{22}$ In the first step of the synthesis, $500 \mu \mathrm{L}$ of $6 \mathrm{ML}$ NPL stock solution was diluted with $5 \mathrm{~mL}$ of hexane in a $15-\mathrm{mL}$ centrifugation tube with a final optical density of $0.2(30 \mu \mathrm{L}$ of the solution diluted with $1 \mathrm{~mL}$ hexane) at the hh-transition maximum. In the next step, $75 \mu \mathrm{L}$ of OlAm, $1 \mathrm{~mL}$ of FA and $75 \mu \mathrm{L}$ of aqueous $\left(\mathrm{NH}_{4}\right)_{2} \mathrm{~S}$ were added. Hexane and FA form a two-phase system in order to separate the ionic precursors from the NPLs to hinder homo-nucleation of CdS. After shaking the tube for $4 \mathrm{~h}$, the hexane phase was separated and washed three times with fresh $1 \mathrm{~mL}$ FA portions. Finally, to complete the shell after the deposition of the sulfur layer, $10 \mu \mathrm{L}$ of fresh OlAm and $25 \mu \mathrm{L}$ of Cd- or $25 \mu \mathrm{L}$ of Zn-precursor stock solutions (see below) diluted with $950 \mu \mathrm{L}$ of FA were added with subsequent shaking for $1 \mathrm{~h}$. The washing steps were repeated. The cation precursor stock solutions were prepared by dissolving $335 \mathrm{mg}$ of $\mathrm{Cd}(\mathrm{OAc})_{2} \cdot 2 \mathrm{H}_{2} \mathrm{O}(1.26 \mathrm{mmol})$ or $276 \mathrm{mg}$ of $\mathrm{Zn}(\mathrm{OAc})_{2} \cdot 2 \mathrm{H}_{2} \mathrm{O}(1.26 \mathrm{mmol})$ in $15 \mathrm{~mL}$ of FA.

\section{Synthesis of 6 ML and 7 ML CdSe NPLs mixture.}

To obtain the next population of the NPLs we modified the 6 ML CdSe NPL synthesis described above. In this approach, directly after the rapid addition of $138.3 \mathrm{mg}$ of $\mathrm{Cd}(\mathrm{OAc})_{2}(0.64 \mathrm{mmol})$ and $81.2 \mathrm{mg}$ of $\mathrm{CdF}_{2}(0.54 \mathrm{mmol})$ at $210^{\circ} \mathrm{C}$ to the reaction mixture, we added $6 \mathrm{~mL}$ of $0.081 \mathrm{M} \mathrm{SeO}_{2}$-in-ODE with a rate of $16 \mathrm{~mL} / \mathrm{h}$. The selenium precursor was prepared by dissolving $54 \mathrm{mg}$ of $\mathrm{SeO}_{2}(0.49 \mathrm{mmol})$ in $6 \mathrm{~mL}$ of $\mathrm{ODE}$ at $200^{\circ} \mathrm{C}$, yielding a clear orange-yellow solution. After the addition, the temperature was raised to $280^{\circ} \mathrm{C}$ and the reaction mixture was kept at this temperature for $40 \mathrm{~min}$. Thereafter, the growth was quenched by rapid cooling with a water bath and the addition of $2 \mathrm{~mL}$ of OlAc at $200^{\circ} \mathrm{C}$. Thus obtained solution was divided into two parts and filled into $50-\mathrm{mL}$ centrifugation tubes, whereto $20 \mathrm{~mL}$ of a hexane:ethanol (3:1) mixture were added with subsequent centrifugation at $4000 \mathrm{rpm}(\mathrm{rcf}=1972 \mathrm{~g})$ for 5 min to separate the NPLs from CdSe nanoparticles typically formed as a byproduct. The precipitate was dissolved in $8 \mathrm{~mL}$ of hexane. To separate large 5 ML from 6 and 7 ML CdSe NPLs we used an additional centrifugation step of the resulting solution at $12000 \mathrm{rpm}$ for $10 \mathrm{~min}$ yielding a supernatant containing a mixture of 6 and 7 ML thick NPLs. 


\section{Influence of Cadmium Halides.}

A) To analyze the impact of the halides on the growth mechanism we modified the synthesis of Ott et al. ${ }^{16}$ via exchange of 10 mol- $\%$ of $\mathrm{Cd}(\mathrm{OH})_{2}$ with $\mathrm{CdX} 2\left(\mathrm{X}=\mathrm{F}, \mathrm{Cl}, \mathrm{Br}, \mathrm{I}, \mathrm{OAc}\right.$, or OAc $\left.\cdot \mathrm{H}_{2} \mathrm{O}\right)$. First, $50 \mathrm{mg}$ of $\mathrm{Cd}(\mathrm{OH})_{2}(0.34 \mathrm{mmol})$ and $27 \mathrm{mg}$ of Se powder $(0.34 \mathrm{mmol})$ in $20 \mathrm{~mL}$ ODE were degassed at $115^{\circ} \mathrm{C}$ for $20 \mathrm{~min}$. Further, the temperature was raised up to $180^{\circ} \mathrm{C}$ under argon atmosphere. To start the reaction, $40 \mu \mathrm{L}$ of valeric acid $(0.34 \mathrm{mmol})$ were injected at $180^{\circ} \mathrm{C}$ and the temperature was kept constant over the complete reaction time. To analyze the impact of the specific halide, $\mathrm{X}$, we used $45 \mathrm{mg}$ of $\mathrm{Cd}(\mathrm{OH})_{2}(0.306 \mathrm{mmol})$ and the corresponding amount of each $\mathrm{CdX}_{2}$ compound $(0.034 \mathrm{mmol})$ in the synthesis.

B) We also tested the influence of $\mathrm{CdCl}_{2}$ on the growth process of CdSe NPLs using the common recipe for 4 ML-thick platelets. For the synthesis of 4 ML CdSe NPLs, $70 \mathrm{mg}$ of CdO (0.54 mmol) and $340 \mathrm{mg}$ of myristic acid $(1.4 \mathrm{mmol})$ in $28 \mathrm{~mL}$ of ODE were degassed at $110^{\circ} \mathrm{C}$ for $30 \mathrm{~min}$ in a 50 -mL three-neck flask. The resulting reddish precursor solution was heated up to $285^{\circ} \mathrm{C}$ under argon atmosphere. After 20 min, the solution become completely transparent and was degassed again at $90^{\circ} \mathrm{C}$ before adding $24 \mathrm{mg}$ of Se powder $(0.3 \mathrm{mmol})$ suspended in $2 \mathrm{~mL}$ of ODE. The reaction mixture was then heated to $240^{\circ} \mathrm{C}$ with the swift addition of $160 \mathrm{mg}$ of $\mathrm{Cd}(\mathrm{OAc})_{2} \cdot 2 \mathrm{H}_{2} \mathrm{O}(0.64 \mathrm{mmol})$ mixed with different amounts of $\mathrm{CdCl}_{2}$ : $11 \mathrm{mg}(0.064 \mathrm{mmol}), 33 \mathrm{mg}(0.192 \mathrm{mmol}), 55 \mathrm{mg}(0.32 \mathrm{mmol}), 99 \mathrm{mg}(0.576 \mathrm{mmol})$, or $110 \mathrm{mg}$ $(0.64 \mathrm{mmol})$ at $190-200^{\circ} \mathrm{C}$. After $10 \mathrm{~min}$ at $240^{\circ} \mathrm{C}$, we quenched the reaction by rapid cooling in a water bath and the addition of $1 \mathrm{~mL}$ of OlAc at $180^{\circ} \mathrm{C}$. The solution was divided into two parts and placed in 50 -mL centrifugation tubes; $20 \mathrm{~mL}$ of a hexane:ethanol (3:1) mixture were added to each portion with subsequent centrifugation at $4000 \mathrm{rpm}(\mathrm{rcf}=1972 \mathrm{~g})$ for $10 \mathrm{~min}$. The precipitate was dissolved in $4 \mathrm{~mL}$ of hexane.

\section{Synthesis of CdS NPLs with and without $\mathrm{CdCl}_{2}$.}

To investigate the effect of halides on the growth of the other cadmium chalcogenide NPLs, we used recipes in the literature to synthesize $\mathrm{CdS}^{23}$ and $\mathrm{CdTe}^{24}$ NPLs. To prepare 4 ML CdS NPLs, $217 \mathrm{mg}$ of $\mathrm{Cd}(\mathrm{OAc})_{2} \cdot 2 \mathrm{H}_{2} \mathrm{O}(0.81 \mathrm{mmol})$ in $0.24 \mathrm{~mL}$ of OlAc and $10 \mathrm{~mL}$ of ODE were stirred in a three-neck flask under nitrogen atmosphere at room temperature for $30 \mathrm{~min}$. Next, $2 \mathrm{~mL}$ of $0.1 \mathrm{M} \mathrm{S-ODE}(0.2 \mathrm{mmol})$ prepared as described above were added as a sulfur precursor at room temperature and stirred for another $10 \mathrm{~min}$. To start the reaction, the mixture was heated up to $260^{\circ} \mathrm{C}$. In order to assess the effect of $\mathrm{CdCl}_{2}$ on the growth of CdS NPLs, we added $14.18 \mathrm{mg}$ of $\mathrm{CdCl}_{2}(0.081 \mathrm{mmol})$ when the temperature of the reaction mixture was raised above $200^{\circ} \mathrm{C}$. After $3 \mathrm{~min}$ at $260^{\circ} \mathrm{C}$, the reaction was quenched to room temperature while $1 \mathrm{~mL}$ OlAc was added at $180^{\circ} \mathrm{C}$. Finally, the NPLs were precipitated by adding 20 
$\mathrm{mL}$ of hexane:ethanol (3:1) mixture to the reaction solution followed by centrifugation at $7000 \mathrm{rpm}$. The precipitate was dissolved in $2 \mathrm{~mL}$ of hexane.

\section{Synthesis of CdTe NPLs with and without $\mathrm{CdCl}_{2}$.}

To synthesize $4 \mathrm{ML}$ CdTe NPLs, $133 \mathrm{mg}$ of Cd(OAc) $)_{2} \cdot 2 \mathrm{H}_{2} \mathrm{O}(0.5 \mathrm{mmol}), 0.255 \mathrm{~mL}$ of OlAc and $25 \mathrm{~mL}$ of ODE were degassed under vacuum in a three-neck flask at $95^{\circ} \mathrm{C}$ for two hours. The temperature was raised to $215^{\circ} \mathrm{C}$ under a nitrogen atmosphere and $22 \mu \mathrm{L}(0.05 \mathrm{mmol}$ of Te) of $2.24 \mathrm{M}$ Te-TOP (prepared in a nitrogen-filled glovebox by dissolving $284.48 \mathrm{mg}$ of Te-powder in $1 \mathrm{~mL}$ of TOP under via stirring for $2 \mathrm{~h}$ at $100^{\circ} \mathrm{C}$ ) diluted with $2.5 \mathrm{~mL}$ of ODE were slowly added to the reaction mixture and then kept at $215^{\circ} \mathrm{C}$ for $15 \mathrm{~min}$. To test the influence of halides, we added $45.75 \mathrm{mg}$ of $\mathrm{CdCl}_{2}(0.25 \mathrm{mmol})$ in $3 \mathrm{~min}$ after the Te-TOP injection and stirred the reaction mixture for additional $15 \mathrm{~min}$ at $215^{\circ} \mathrm{C}$. To quench the reaction, the system was quickly cooled down while $1 \mathrm{~mL}$ of OlAc was added at $180^{\circ} \mathrm{C}$. The mixture was then divided into two parts, filled in $50-\mathrm{mL}$ centrifugation tubes and then $20 \mathrm{~mL}$ of a hexane:ethanol (3:1) mixture was added to each part followed by centrifugation at $4000 \mathrm{rpm}$ for $5 \mathrm{~min}$. Finally, the precipitate was dissolved in $4 \mathrm{~mL}$ of hexane.

\section{Characterization.}

Absorption spectra of the NPLs were acquired at room temperature using a Cary 60 UV-Visspectrophotometer (Agilent Technologies) from their diluted solutions in 1-cm-path quartz cuvettes. $\boldsymbol{P L}$ and $\boldsymbol{P L}$ excitation (PLE) spectra were recorded on a Fluoromax-4 spectrofluorometer (Horiba Jobin Yvon Inc.). To estimate the absolute PLQYs we used a FluoroLog-3 spectrofluorometer (Horiba Jobin Yvon Inc.) equipped with a Quanta- $\varphi$ integrating sphere. For all PL and PLQY measurements, an excitation wavelength of $450 \mathrm{~nm}$ was set. Time-resolved $\boldsymbol{P L}$ measurements were performed using a FluoroLog-3 spectrofluorometer (Horiba Jobin Yvon) equipped with a pulsed laser diode (410 nm) and a time-correlated single-photon-counting (TCSPC) module at room temperature. Average PL lifetimes were calculated when the initial signal intensity was reduced to 10000 counts/e. Transmission electron microscopy (TEM) was performed on an FEI Tecnai F30 microscope operated at $300 \mathrm{kV}$ and equipped with an energy dispersive X-ray spectrometer EDAX EDAMIII. CdSe NPLs were drop-cast from their diluted solutions in hexane onto carbon-coated copper grids. High resolution TEM imaging was carried out on a JEOL JEM-2200FS microscope equipped with a Schottky emitter operated at $200 \mathrm{kV}$, a CEOS spherical aberration corrector for the objective lens, and an in-column energy filter (Omega-type). Powder X-ray diffraction (XRD) patterns were collected with a Bruker AXS D2 PHASER diffraction 
system in the reflection mode. A nickel filter, $\mathrm{Cu} \mathrm{K \alpha 1}$ irradiation, and a LYNXEYE/SSD160 detector were used.

\section{THEORETICAL SECTION}

Density-functional theory calculations. We calculated the absolute surface energy of Cd-terminated $\mathrm{CdSe}(001)$ facets passivated by $\mathrm{Cl}$ atoms using density-functional theory (DFT) in the generalizedgradient approximation of Perdew, Burke, and Ernzerhof, as implemented in the Vienna Ab-initio Simulation Package (VASP) ${ }^{25,26}$. The facets were represented by infinite flat surfaces using a periodic slab geometry with 13 atomic layers of $\mathrm{Cd}$ and Se and a vacuum region of $10 \AA$. We found that the $\mathrm{Cl}$ atoms prefer to occupy the bridge site between two $\mathrm{Cd}$ atoms with a coverage of $1 \mathrm{ML}$. The surface energy, $E_{A}$, depends on the $\mathrm{Cl}$ chemical potential, which we assumed to have the maximum value allowed by thermodynamic equilibrium between $\mathrm{CdSe}$ and $\mathrm{CdCl}_{2}$. The surface Brillouin zone was sampled using an $8 \times 8$ Monkhorst-Pack mesh. The plane-wave cutoff was $400 \mathrm{eV}$. All atomic positions were relaxed until the surface energy was converged to within $0.1 \mathrm{meV} / \AA^{2}$.

\section{RESULTS AND DISCUSSION}

TEM images of CdSe NPLs obtained using cadmium fluoride as a growth promoter show rectangular platelets with an average lateral size of $15 \times 10 \mathrm{~nm}$ (Figure 1a). Some of the NPLs form small stacks, allowing the direct determination of their thickness, $2.03 \mathrm{~nm}$, from HRTEM imaging (Figure 1b). Figure $1 \mathrm{c}, \mathrm{d}$ displays optical properties of $6 \mathrm{ML}$ CdSe NPLs obtained by adding $90 \%$ of $\mathrm{CdF}_{2}$ relative to $\mathrm{Cd}(\mathrm{OAc})_{2}$. Heavy hole (hh) and light hole (lh) transitions in absorption spectrum coincide well with those reported for c-ALD synthesized NPLs, $580 \mathrm{~nm}\left(2.13 \mathrm{eV}\right.$ vs. $\left.2.12 \mathrm{eV}^{14}\right)$ and $549 \mathrm{~nm}(2.25 \mathrm{eV}$ vs. 2.24 $\mathrm{eV}^{14}$ ), respectively. The PL spectrum is peaked at $582 \mathrm{~nm}$ with FWHM of approx. $11 \mathrm{~nm}$. Time-resolved PL measurements provide an average PL lifetime of 5.3 ns (Figure 1d) with PLQY reaching 50\%. The PLE spectrum, recorded at $582 \mathrm{~nm}$, resembles the absorption spectrum of the NPLs thus revealing a single population of the $6 \mathrm{ML}$ species in solution. The observed slight deviations of the peak positions are caused by the lateral confinement effects. ${ }^{16,27}$ For example, smaller NPLs $(8 \times 6 \mathrm{~nm})$ synthesized at lower reaction temperature of $\left(260^{\circ} \mathrm{C}\right.$ instead of $\left.280^{\circ} \mathrm{C}\right)$ exhibit the hh-transition shifted to shorter wavelengths by $9 \mathrm{~nm}$ (Figure SI1 in the Supporting Information). As follows from the XRD pattern, addition of halides does not affect the zinc blende crystal structure of the NPLs (Figure 1e). 


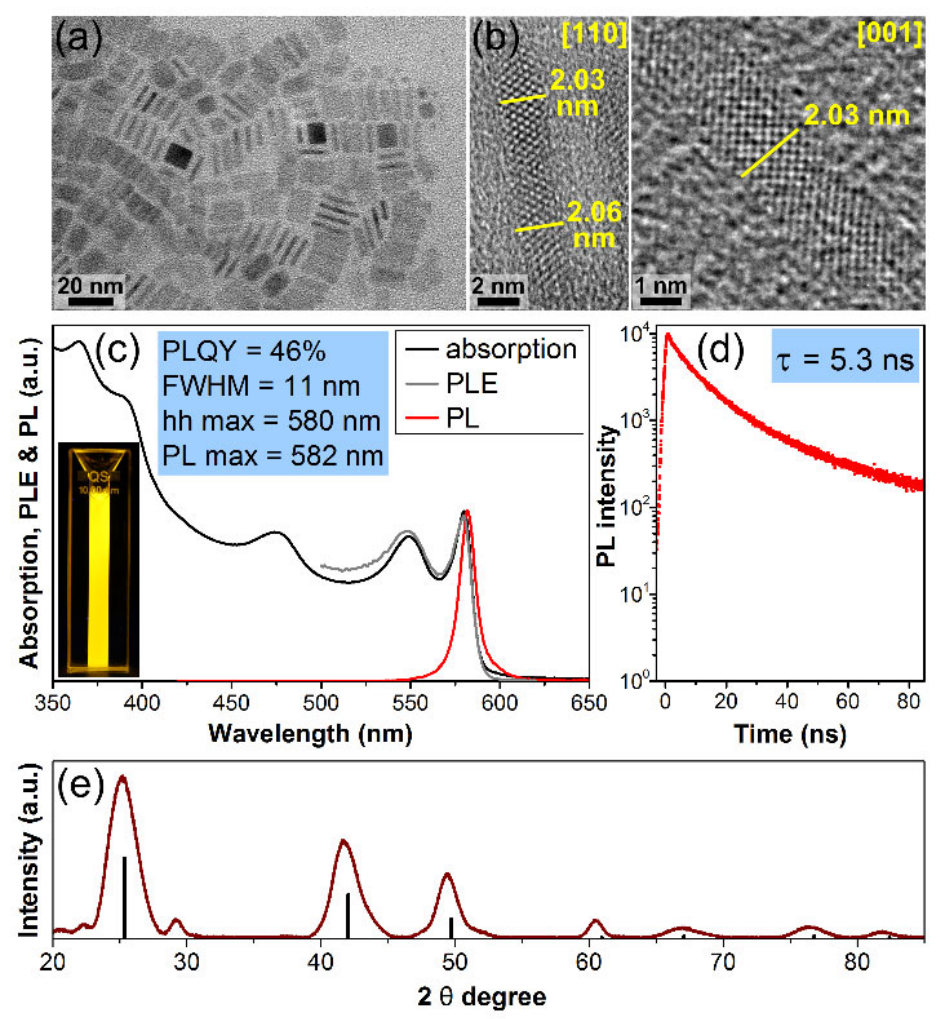

Figure 1. Conventional TEM image of 6ML CdSe NPLs (a). HRTEM images of vertically oriented NPLs (b). Optical properties of 6 ML CdSe NPLs: absorption, PL, PLE spectra (c), and time-resolved PL decay (d). Inset in (c) is a photo of the NPLs in solution under UV-light excitation. XRD pattern of 6 ML CdSe NPLs compared to ICDD reference of cubic CdSe (C19-191) (e).

We observed the formation of metallic $\mathrm{Cd}$ nanoparticles upon heating the mixture of $\mathrm{CdO}$ and myristic acid in $\mathrm{ODE}$ at $305^{\circ} \mathrm{C}$, which appeared as a grey-greenish turbid dispersion. We assume that this reaction is the first key point that changes the nucleation and the growth pathway and allows for the subsequent formation of the thicker NPLs. As seen from TEM images in Figure 2a, cadmium crystallized in both small (few $\mathrm{nm}$ ) and large (more than $100 \mathrm{~nm}$ ) nanoparticles. The XRD pattern of the separated product matches well to metallic $\mathrm{Cd}$ and $\mathrm{CdO}$ phases. $\mathrm{CdO}$ can be represented either by unreacted precursor or by (partially) oxidized metallic Cd nanoparticles upon their exposure to air during the measurement. We presume that these metallic nanoparticles may possess a higher reactivity than $\mathrm{Cd}$ carboxylate changing the energetic balance, in particular activation energy barrier, in the subsequent reaction with Se-precursor. The melting point of the bulk $\mathrm{Cd}$ is $321^{\circ} \mathrm{C},{ }^{28}$ which may be significantly depressed by decreasing the size of the metal to few nanometers. Thus, in the reaction mixture these particles can exist in the form of melt acting as a $\mathrm{Cd}$ reservoir, thus eventually changing the concentration of active $\mathrm{Cd}$ species. 


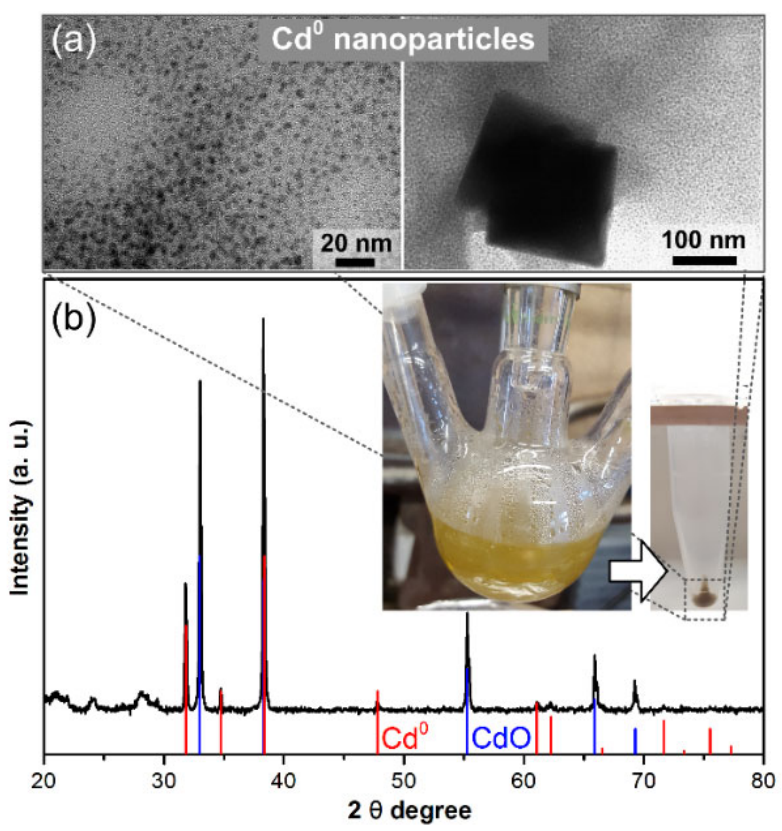

Figure 2. TEM images of small and large $\mathrm{Cd}^{0}$ nanoparticles (a). XRD pattern of the precipitate sampled at the beginning of the NPLs synthesis from the grey/green turbid solution along with $\mathrm{Cd}(\mathrm{C} 5-674)$ and $\mathrm{CdO}(\mathrm{C} 5-640)$ references (b). In the inset are photos of the reaction mixture containing $\mathrm{Cd}(\mathrm{CdO})$ nanoparticles and the corresponding precipitate.

The second key modification of the NPL synthesis is the use of cadmium halides as a part of Cdprecursor. The best result was obtained by employing $84 \%-$ mol. of $\mathrm{CdF}_{2}$ relative to $\mathrm{Cd}(\mathrm{OAc})_{2}$. This mixture, in the form of a solid powder, was quickly added to the preformed Cd nanoparticles with Se dispersed in ODE at $280^{\circ} \mathrm{C}$. Generally, halides (both inorganic species and organic molecules) play an important role in many colloidal syntheses, such as the preparation of $\mathrm{InP}^{29}$ and $\mathrm{GaAs}^{30}$ nanoparticles, $\mathrm{CdSe} / \mathrm{CdS}$ octapods, ${ }^{31}$ and $\mathrm{PbS}^{32}$ and $\mathrm{CdSe}^{33}$ nanosheets. ${ }^{34}$ We tested all cadmium halides in the synthesis of CdSe NPLs and obtained the best and reproducible results with $\mathrm{CdF}_{2}$. To demonstrate the impact of these additives, we adopted the synthesis recipe published by Ott et al., ${ }^{16}$ used to study the NPL ripening over long reaction times. We employed several $\mathrm{Cd}$ precursors, such as pure $\mathrm{Cd}(\mathrm{OH})_{2}$ and 9:1-mol. mixture of $\mathrm{Cd}(\mathrm{OH})_{2}$ with $\mathrm{Cd}(\mathrm{OAc})_{2}, \mathrm{CdF}_{2}, \mathrm{CdCl}_{2}, \mathrm{CdBr}_{2}$, or $\mathrm{CdI}_{2}$. The progress of the reaction was monitored by absorption spectroscopy, which clearly indicated the formation of each population of the NPLs: 2 ML (hh transition at $395 \mathrm{~nm}$ ), $3 \mathrm{ML}$ (463 nm), and $4 \mathrm{ML}$ (510 nm) thick species (Figure 3). Analysis of the spectra reveal that, in the case of $\mathrm{Cd}(\mathrm{OH})_{2}$, mainly 2 ML NPLs formed during $250 \mathrm{~min}$ of the ripening, whereas $\mathrm{Cd}(\mathrm{OAc})_{2}$ and $\mathrm{Cd}(\mathrm{OAc})_{2} \cdot 2 \mathrm{H}_{2} \mathrm{O}$ promoted a much faster growth and led to the formation of 3 ML NPLs and small fraction of 4 ML NPLs. Addition of the halides further accelerated 
the ripening, yielding 4 ML NPLs under the same reaction conditions. Among the three additives tested, $\mathrm{CdCl}_{2}$ was the most active promotor of $4 \mathrm{ML}$ platelets from the very beginning of the ripening. All spectra showed a small red shift of the main peaks when NPLs appeared and grew, which then blueshifted when they decomposed and transformed to the next population. We note that when employing $\mathrm{CdF}_{2}$ we obtained highly turbid solutions which complicated absorption measurements. In this series of halides, nucleophilicity (in aprotic solvents) and Lewis basicity increase from iodide to fluoride. ${ }^{35}$ Hence, a stronger base is needed to effectively suppress the activation energy barrier of formation of the next CdSe ML.

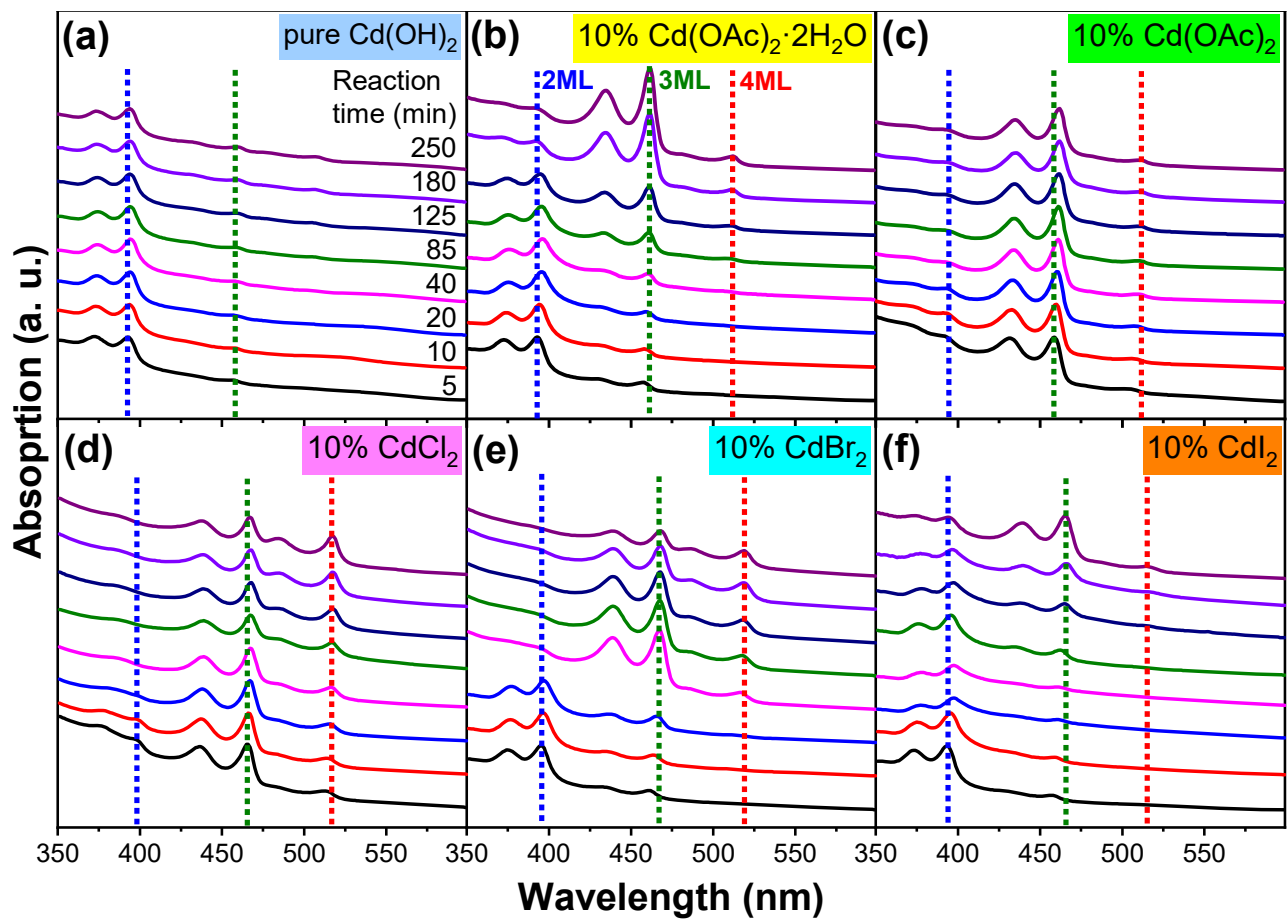

Figure 3. Evolution of the absorption spectra of CdSe NPLs grown at $180^{\circ} \mathrm{C}$ using: pure $\mathrm{Cd}(\mathrm{OH})_{2}(\mathrm{a})$, 9:1-mol. mixture of $\mathrm{Cd}(\mathrm{OH})_{2}$ with $\mathrm{Cd}(\mathrm{OAc})_{2}$ (b), $\mathrm{Cd}(\mathrm{OAc})_{2} \cdot 2 \mathrm{H}_{2} \mathrm{O}$ (c), $\mathrm{CdCl}_{2}$ (d), $\mathrm{CdBr}_{2}$ (e), and $\mathrm{CdI}_{2}$ (f). Distinct absorption features of $2 \mathrm{ML}(395 \mathrm{~nm}), 3 \mathrm{ML}(463 \mathrm{~nm})$, and 4 ML (510 nm) NPLs are indicated by dashed vertical lines.

We also tested the impact of the amount of $\mathrm{CdCl}_{2}$ added to $\mathrm{Cd}(\mathrm{OAc})_{2} \cdot 2 \mathrm{H}_{2} \mathrm{O}$ on the growth of $\mathrm{CdSe}$ NPLs, using the common recipe for 4 ML platelets. Figure 4 displays the evolution of the shape and the size of the resulting NPLs; optical spectra are presented in Figure SI2. Combining both sets of data, we see that upon increasing the amount of $\mathrm{CdCl}_{2}$, the population of $5 \mathrm{ML}$ thick NPLs increases. By adding $70 \%$ of $\mathrm{CdCl}_{2}$ (relative to $\mathrm{Cd}(\mathrm{OAc})_{2}$ ) we obtained solely $5 \mathrm{ML}$ NPLs, as follows from the absence of optical features from thinner NPLs in the spectra. Lower concentrations of the promoter (10-50\%) led 
to the formation of two distinctly different fractions of the NPLs, rounded disk-like and rectangular platelets. Presumably, the disks are 4 ML NPLs whereas the rectangles represent 5 ML NPLs, since in the samples containing solely 5 ML NPLs only rectangular shaped platelets are present. In addition to the growth in z-direction, the NPLs extend in lateral directions reaching an average size of $20 \times 60 \mathrm{~nm}$ with $90 \%$ of $\mathrm{CdCl}_{2}$. Using $100 \%$ of $\mathrm{CdCl}_{2}\left(1: 1-\mathrm{mol}\right.$ mixture with $\left.\mathrm{Cd}(\mathrm{OAc})_{2}\right)$ yielded strongly etched NPLs with decreased lateral sizes, while their thickness was preserved at 5 MLs. Thus, the addition of cadmium halides not only facilitates the nucleation of the next CdSe ML but also promotes the growth of the NPLs in the lateral dimensions.

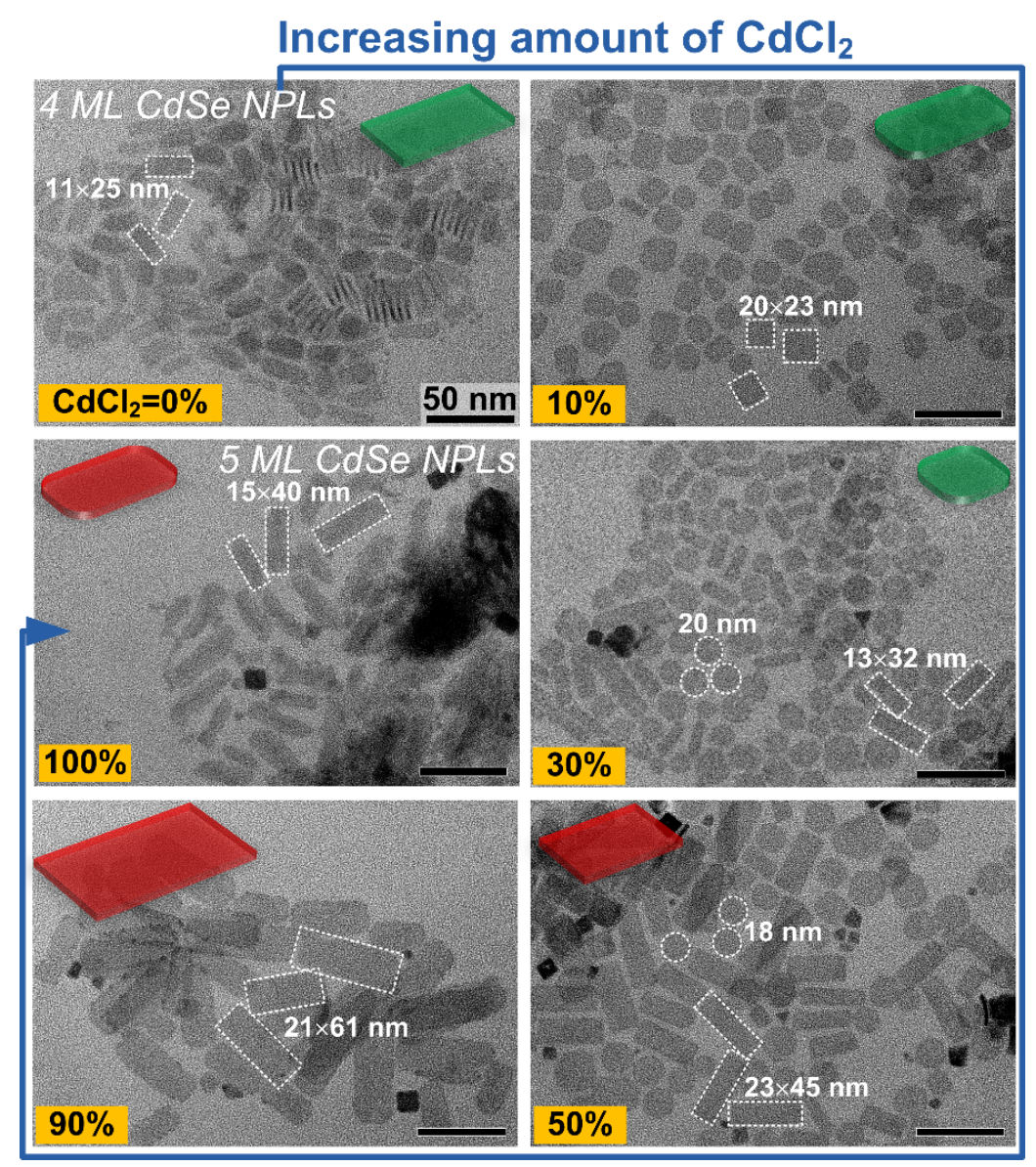

Figure 4. TEM images of CdSe NPLs obtained with different amounts of $\mathrm{CdCl}_{2}(0,10,30,50,90$, or $100 \%$ relative to cadmium acetate) using recipe for 4 ML NPLs synthesis, showing the evolution of the size and the shape of the resulting particles.

In order to better understand the role of halides, we turn to the NPL growth model proposed by Riedinger et al. ${ }^{15}$ and focus on one particular promoter, $\mathrm{CdCl}_{2}$. The addition of $\mathrm{CdCl}_{2}$ to the synthesis can affect the growth kinetics in two ways. First, if the binding of $\mathrm{Cl}$ atoms to $\mathrm{Cd}$ atoms on the surface 
of the CdSe NPL is more favorable than the binding of acetate ligands, then the $\mathrm{Cl}$ atoms may displace the organic ligands. This change will reduce the surface energy of the NPLs and, according to the growth model in ref. ${ }^{15}$, will alter the energy landscape governing the nucleation of islands during growth. Cho et al. ${ }^{19}$ also analyzed this effect and pointed out its somewhat counterintuitive consequence: that decreasing the surface energy leads not to thicker NPLs but to thinner ones. They also pointed out a second consequence: the addition of $\mathrm{Cl}$ also affects (here, by reducing) the enthalpy of the reaction that produces CdSe from its precursors. According to the growth model, decreasing this volume energy indeed leads to thicker NPLs, consistent with their experimental results. As a guide for understanding the general outcome of these two competing effects, we show in Figure 5 the theoretically predicted maximum stable thickness of NPLs as a function of the surface energy, $E_{A}$, and volume energy (equivalent to the reaction enthalpy), $E_{V}$, according to the model of Riedinger et al. ${ }^{15}$. (The model also depends on the energy per unit length $E_{L}$ along a step, for simplicity assumed here constant.) The results of Riedinger et al. ${ }^{15}$ (maximum thickness $5 \mathrm{ML}$ ) are denoted by the white circle and the results of Cho et al. ${ }^{19}$ (maximum thickness $6 \mathrm{ML}$ ) by the yellow circle. For the latter case we computed $E_{A}$ for Clpassivated CdSe NPLs using DFT and obtained the value $5.1 \mathrm{meV} / \AA^{2}$, as shown. The value of $E_{V}$ was derived in the work of Cho et al. ${ }^{19}$ on the basis of standard reaction enthalpies combined with an experimental measurement of the amount of $\mathrm{Cl}$ present in their synthesis. For understanding the present work, it is important to note that both $E_{A}$ and $E_{V}$ depend on the specific halide and so no general conclusions can be drawn. For the specific case of $\mathrm{Cl}, E_{A}$ and $E_{V}$ must have the same values as in the work of Cho et al. ${ }^{19}$ but the fractional amount of $\mathrm{Cl}$ in the synthesis may be different. If this amount is larger then $E_{V}$ would be further reduced in magnitude. The consequence would be even thicker stable NPLs, which would be consistent with our experimental findings below. 


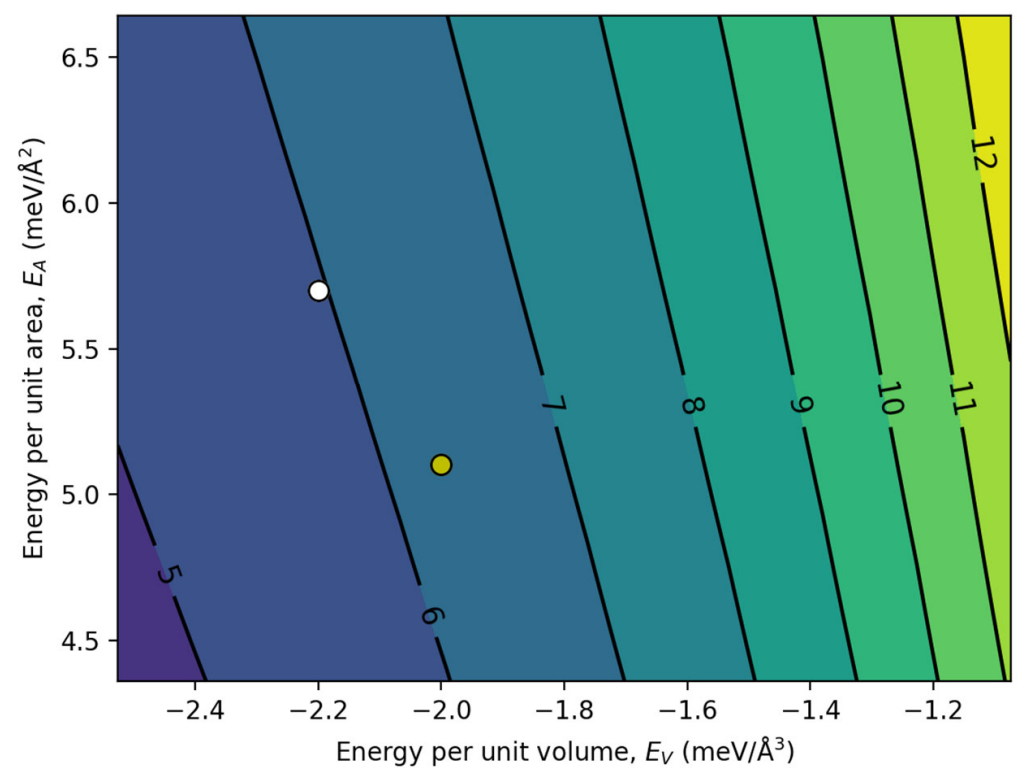

Figure 5. Theoretically predicted maximum stable thickness in MLs of CdSe NPLs according to the growth model proposed by Riedinger et al. ${ }^{15}$ The white circle denotes the values of $E_{V}$ and $E_{A}$ obtained in that work for CdSe NPLs passivated by acetate molecules, which leads to NPLs with maximum thickness of $5 \mathrm{ML}$. The yellow circle denotes the results of Cho et al. ${ }^{19}$ for CdSe NPLs passivated by $\mathrm{Cl}$ atoms, which leads to NPLs with maximum thickness of 6 ML.

In addition, we explored syntheses of $\mathrm{CdS}^{23}$ and $\mathrm{CdTe}^{24}$ NPLs and repeated the experiments with the addition of small amounts of cadmium chloride to underline the general impact of halides on the growth process of all cadmium chalcogenide-based NPLs. According to the optical spectroscopy data presented in Figure SI3a,b, the addition of $10 \%$ of $\mathrm{CdCl}_{2}$ (relative to cadmium acetate) in the modified synthesis of 4 ML CdS NPLs resulted in a red shift in the absorption spectrum (from $414 \mathrm{~nm}$ to $433 \mathrm{~nm}$ ) and the PL spectrum (from $419 \mathrm{~nm}$ to $435 \mathrm{~nm}$ ), indicating the formation of a large fraction of $5 \mathrm{ML} \mathrm{CdS}$ NPLs instead of 4 ML thick platelets growing without addition of the promoter. The shape of both populations of the NPLs was quite irregular and the size distribution rather broad, and hence did not allow for a proper comparison of the morphological changes of the platelets obtained with and without $\mathrm{CdCl}_{2}$ (Figure SI3c,d). In the case of CdTe NPLs synthesis, the impact of $\mathrm{CdCl}_{2}$ was not so pronounced. Its addition did not lead to the complete transformation from 3 ML CdTe NPLs (absorption maximum at $502 \mathrm{~nm}$, PL maximum at $504 \mathrm{~nm}$ ) to thicker platelets (Figure SI4a,b). Nevertheless, one may see additional small features at $557 \mathrm{~nm}, 599 \mathrm{~nm}$, and $632 \mathrm{~nm}$ in the absorption spectrum of the platelets grown in the presence of $\mathrm{CdCl}_{2}$ corresponding to the next populations, i.e. $4 \mathrm{ML}, 5 \mathrm{ML}$, and $6 \mathrm{ML} \mathrm{CdTe}$ NPLs, respectively ${ }^{14}$. Appearance of much more distinct additional peaks at $560 \mathrm{~nm}, 601 \mathrm{~nm}$, and 635 
$\mathrm{nm}$ in the PL spectrum further indicates the formation of the thicker platelets. From the TEM images we observe distinctly different shapes for NPLs grown with $\mathrm{CdCl}_{2}$, rounded rather than square or rectangular ones, compared to those synthesized without the addition of chloride (Figure SI4c,d). Therefore, we may assume a general character of the impact of halides on the growth of 2D nanostructures built of all three cadmium chalcogenides.

We also used the obtained $6 \mathrm{ML}$ CdSe NPLs to synthesize core/crown and core/shell CdSe/CdS heterostructured NPLs. Core/crown NPLs exhibited a small red shift of their absorption and PL spectra compared to just core NPLs, while in the case of core/shell structure the red shift was much more evident, as can be expected (Figure 6). The PLQYs of these samples were found to be $44 \%$ and $36 \%$ for the core/crown and the core/shell structures, respectively. Both heterostructures have short average PL lifetimes comparable with that of only-core NPLs: $3.9 \mathrm{~ns}$ for the core/crown and $6.4 \mathrm{~ns}$ for the core/shell NPLs (vs. $5.3 \mathrm{~ns}$ for the core only) (Figure 6c). The growth of the CdS crown is indicated by both absorption spectroscopy and TEM imaging (Figure 6a,e). In the first case, one can observe a strong absorption in the blue region attributed to nanostructured CdS. In the latter, increased size of the NPLs and their irregular shape are direct proof of the core growth. At the same time, the shell growth did not lead to a remarkable change of the shape and the lateral size of the core CdSe NPLs, as seen in the TEM image of Figure $6 f$. 

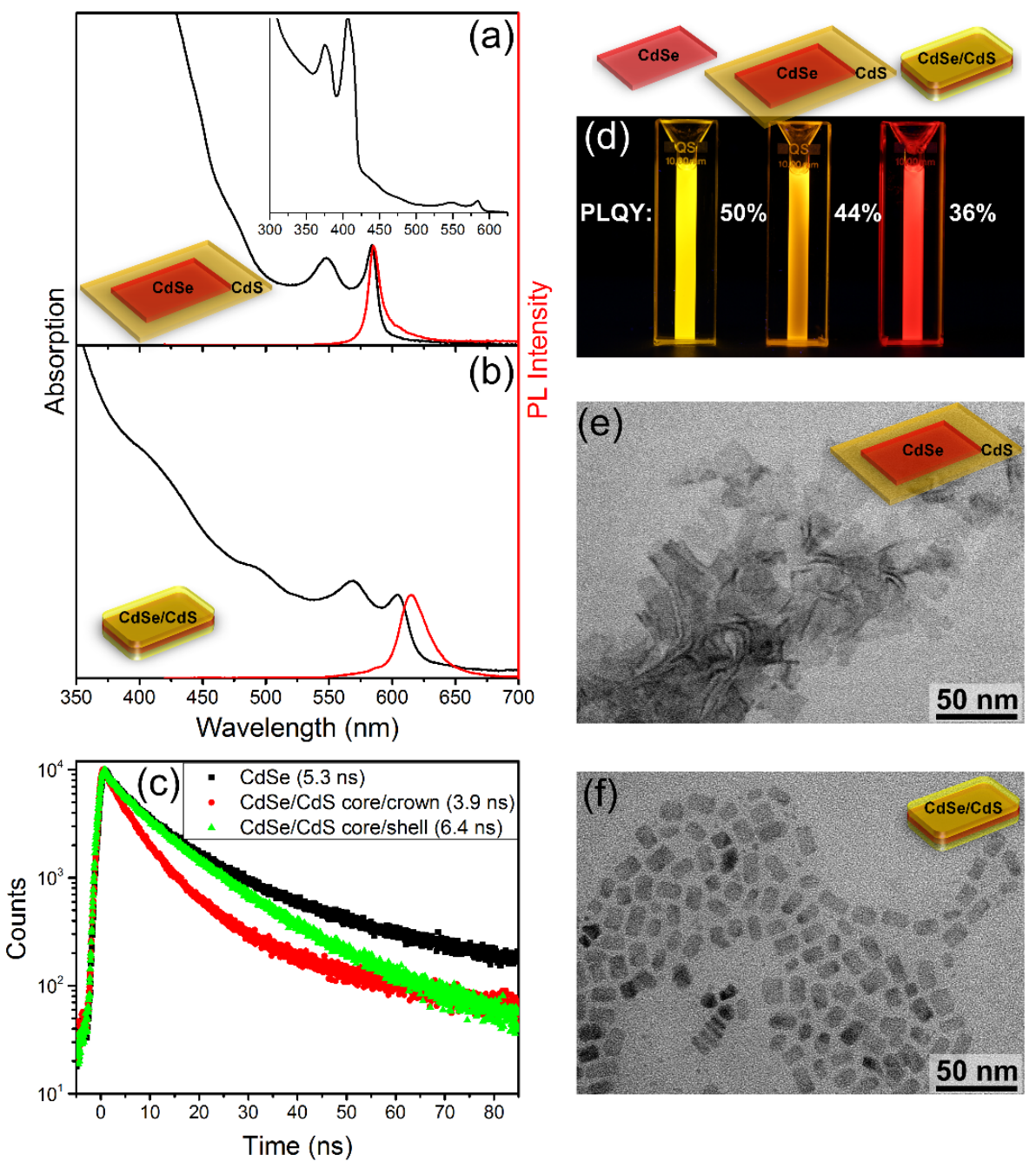

Figure 6. Optical properties of core/crown and core/shell 6 ML CdSe/CdS NPLs: absorption, steady state PL (a, b), and time resolved PL spectra (c). Photo of $6 \mathrm{ML}$ CdSe core, core/crown, and core/shell $\mathrm{CdSe} / \mathrm{CdS}$ NPL colloids under UV-light excitation (d). TEM images of core/crown (e) and core/shell (f) $\mathrm{CdSe} / \mathrm{CdS}$ NPLs.

By attempting the seeded growth of $6 \mathrm{ML}$ CdSe NPLs in order to increase their lateral size, we observed the appearance of 7 ML thick particles, as evidenced by the absorption and PL spectra in Figure SI5a. Additional to 6 ML NPLs, the hh-transition at $596 \mathrm{~nm}$ and pronounced sharp PL peaked at $601 \mathrm{~nm}$ indicate the formation of a significant fraction of 7 ML CdSe NPLs. From TEM images of this sample, one can distinguish two different populations of the platelets according to their size and contrast: larger rectangular species which we attribute to 6 ML NPLs, and smaller particles which appear as darker squares and which we attribute to 7 ML NPLs (Figure SI5b). The emission maximum at $601 \mathrm{~nm}$ matches the data of Cho et al. ${ }^{19}$, which they attributed to the next population of CdSe NPLs. 


\section{CONCLUSIONS}

We studied the impact of cadmium halides on the growth of colloidal 2D cadmium sulfide, selenide, and telluride nanocrystals. In all three cases, the addition of halides yielded thicker NPLs. Cadmium fluoride and chloride were found to be the most active promoters of this growth on the example of CdSe NPLs, which resulted in the pure population of $6 \mathrm{ML}$ thick species. Moreover, attempting the seeded growth of these NPLs in order to increase their lateral dimensions we observed the formation of even thicker, 7 ML, platelets. This underlines the potential of cadmium halides for controlling the growth kinetics of these $2 \mathrm{D}$ nanocrystals, as we demonstrated by explicitly calculating the surface energy of CdSe in the presence of chloride ligands. Another important modification of the synthetic procedure was the formation of metallic $\mathrm{Cd}$ nanoparticles at the stage of the Cd-precursor preparation, which can serve as additional cadmium reservoirs in the reaction mixture. In addition to increasing the thickness of the NPLs by one extra CdSe monolayer, it was also possible to extend their lateral dimensions by using cadmium halides. The resulting atomic flat nanocrystals showed optical properties typical for CdSe NPLs: extremely narrow emission spectra with FWHM of approx. $11 \mathrm{~nm}$ centered at $582 \mathrm{~nm}$, small Stokes shift of $2 \mathrm{~nm}$, high PLQY of up to 50\% and a short PL lifetime about $5 \mathrm{~ns}$. Using these NPLs as cores, we also synthesized core/shell and core/crown $\mathrm{CdSe} / \mathrm{CdS}$ heterostructured platelets. Our findings pave the way to the direct synthesis of thicker NPLs possessing excellent optical properties suitable for optoelectronic applications, such as light emitting diodes and lasing.

\section{ASSOCIATED CONTENT}

\section{Supporting Information}

Experimental methods; additional absorption and PL spectra; TEM images of CdSe NPLs synthesized at lower temperature; absorption and PL spectra, as well as TEM images of CdS and CdTe NPLs.

\section{AUTHOR INFORMATION}

\section{Corresponding Author}

*E-mail: vladimir.lesnyak@chemie.tu-dresden.de.

\section{ACKNOWLEDGMENT}

S.C.E. acknowledges support by the U.S. Office of Naval Research through the Naval Research Laboratory's Basic Research Program. Computations were performed at the DoD Major Shared Resource Center and AFRL. This work was supported by the International Excellence Graduate School on 
Emerging Materials and Processes Korea (iEGSEMP Korea) in the context of TU Dresden's Institutional Strategy The Synergetic University, by the DAAD project 57334961, and by the EU Horizon 2020 project MiLEDi (779373).

\section{REFERENCES}

1. Bouet, C.; Tessier, M. D.; Ithurria, S.; Mahler, B.; Nadal, B.; Dubertret, B., Flat Colloidal Semiconductor Nanoplatelets. Chem. Mater. 2013, 25, 1262-1271.

2. Lhuillier, E.; Pedetti, S.; Ithurria, S.; Nadal, B.; Heuclin, H.; Dubertret, B., Two-Dimensional Colloidal Metal Chalcogenides Semiconductors: Synthesis, Spectroscopy, and Applications. Acc. Chem. Res. 2015, 48, 22-30.

3. Nasilowski, M.; Mahler, B.; Lhuillier, E.; Ithurria, S.; Dubertret, B., Two-Dimensional Colloidal Nanocrystals. Chem. Rev. 2016, 116, 10934-10982.

4. Ithurria, S.; Tessier, M. D.; Mahler, B.; Lobo, R. P. S. M.; Dubertret, B.; Efros, A. L., Colloidal Nanoplatelets with Two-Dimensional Electronic Structure. Nat. Mater. 2011, 10, 936-941.

5. Yeltik, A.; Delikanli, S.; Olutas, M.; Kelestemur, Y.; Guzelturk, B.; Demir, H. V., Experimental Determination of the Absorption Cross-Section and Molar Extinction Coefficient of Colloidal CdSe Nanoplatelets. J. Phys. Chem. C 2015, 119, 26768-26775.

6. Achtstein, A. W.; Antanovich, A.; Prudnikau, A.; Scott, R.; Woggon, U.; Artemyev, M., Linear Absorption in CdSe Nanoplates: Thickness and Lateral Size Dependency of the Intrinsic Absorption. $J$. Phys. Chem. C 2015, 119, 20156-20161.

7. Olutas, M.; Guzelturk, B.; Kelestemur, Y.; Yeltik, A.; Delikanli, S.; Demir, H. V., Lateral SizeDependent Spontaneous and Stimulated Emission Properties in Colloidal CdSe Nanoplatelets. ACS Nano 2015, 9, 5041-5050.

8. Guzelturk, B.; Kelestemur, Y.; Olutas, M.; Delikanli, S.; Demir, H. V., Amplified Spontaneous Emission and Lasing in Colloidal Nanoplatelets. ACS Nano 2014, 8, 6599-6605.

9. She, C.; Fedin, I.; Dolzhnikov, D. S.; Demortière, A.; Schaller, R. D.; Pelton, M.; Talapin, D. V., Low-Threshold Stimulated Emission Using Colloidal Quantum Wells. Nano Lett. 2014, 14, 2772-2777. 10. Lhuillier, E.; Robin, A.; Ithurria, S.; Aubin, H.; Dubertret, B., Electrolyte-Gated Colloidal Nanoplatelets-Based Phototransistor and Its Use for Bicolor Detection. Nano Lett. 2014, 14, 2715-2719. 11. Lhuillier, E.; Pedetti, S.; Ithurria, S.; Heuclin, H.; Nadal, B.; Robin, A.; Patriarche, G.; Lequeux, N.; Dubertret, B., Electrolyte-Gated Field Effect Transistor to Probe the Surface Defects and Morphology in Films of Thick CdSe Colloidal Nanoplatelets. ACS Nano 2014, 8, 3813-3820. 
12. Kunneman, L. T.; Schins, J. M.; Pedetti, S.; Heuclin, H.; Grozema, F. C.; Houtepen, A. J.; Dubertret, B.; Siebbeles, L. D. A., Nature and Decay Pathways of Photoexcited States in CdSe and CdSe/CdS Nanoplatelets. Nano Lett. 2014, 14, 7039-7045.

13. Rossinelli, A. A.; Riedinger, A.; Marques-Gallego, P.; Knusel, P. N.; Antolinez, F. V.; Norris, D. J., High-Temperature Growth of Thick-Shell CdSe/CdS Core/Shell Nanoplatelets. Chem. Commun. 2017, 53, 9938-9941.

14. Chu, A.; Livache, C.; Ithurria, S.; Lhuillier, E., Electronic Structure Robustness and Design Rules for 2D Colloidal Heterostructures. J. Appl. Phys. 2018, 123, 035701.

15. Riedinger, A.; Ott, F. D.; Mule, A.; Mazzotti, S.; Knusel, P. N.; Kress, S. J. P.; Prins, F.; Erwin, S. C.; Norris, D. J., An Intrinsic Growth Instability in Isotropic Materials Leads to Quasi-TwoDimensional Nanoplatelets. Nat. Mater. 2017, 16, 743-748.

16. Ott, F. D.; Riedinger, A.; Ochsenbein, D. R.; Knüsel, P. N.; Erwin, S. C.; Mazzotti, M.; Norris, D. J., Ripening of Semiconductor Nanoplatelets. Nano Lett. 2017, 17, 6870-6877.

17. Ithurria, S.; Talapin, D. V., Colloidal Atomic Layer Deposition (c-ALD) using Self-Limiting Reactions at Nanocrystal Surface Coupled to Phase Transfer between Polar and Nonpolar Media. J. Am. Chem. Soc. 2012, 134, 18585-18590.

18. Christodoulou, S.; Climente, J. I.; Planelles, J.; Brescia, R.; Prato, M.; Martín-García, B.; Khan, A. H.; Moreels, I., Chloride-Induced Thickness Control in CdSe Nanoplatelets. Nano Lett. 2018, 18, 6248-6254.

19. Cho, W.; Kim, S.; Coropceanu, I.; Srivastava, V.; Diroll, B. T.; Hazarika, A.; Fedin, I.; Galli, G.; Schaller, R. D.; Talapin, D. V., Direct Synthesis of Six-Monolayer (1.9 nm) Thick Zinc-Blende CdSe Nanoplatelets Emitting at $585 \mathrm{~nm}$. Chem. Mater. 2018, 30, 6957-6960.

20. Kolny-Olesiak, J.; Kloper, V.; Osovsky, R.; Sashchiuk, A.; Lifshitz, E., Synthesis and characterization of brightly photoluminescent CdTe nanocrystals. Surf. Sci. 2007, 601, 2667-2670.

21. Kodanek, T.; Banbela, H. M.; Naskar, S.; Adel, P.; Bigall, N. C.; Dorfs, D., Phase Transfer of 1and 2-Dimensional Cd-Based Nanocrystals. Nanoscale 2015, 7, 19300-19309.

22. Meerbach, C.; Tietze, R.; Voigt, S.; Sayevich, V.; Dzhagan, V. M.; Erwin, S. C.; Dang, Z.; Selyshchev, O.; Schneider, K.; Zahn, D. R. T.; Lesnyak, V.; Eychmüller, A., Brightly Luminescent Core/Shell Nanoplatelets with Continuously Tunable Optical Properties. Adv. Optical Mater. 2019, 1801478 .

23. Diroll, B. T.; Talapin, D. V.; Schaller, R. D., Violet-to-Blue Gain and Lasing from Colloidal CdS Nanoplatelets: Low-Threshold Stimulated Emission Despite Low Photoluminescence Quantum Yield. ACS Photonics 2017, 4, 576-583. 
24. Pedetti, S.; Nadal, B.; Lhuillier, E.; Mahler, B.; Bouet, C.; Abécassis, B.; Xu, X.; Dubertret, B., Optimized Synthesis of CdTe Nanoplatelets and Photoresponse of CdTe Nanoplatelets Films. Chem. Mater. 2013, 25, 2455-2462.

25. Kresse, G.; Hafner, J., Ab Initio Molecular Dynamics for Liquid Metals. Phys. Rev. B 1993, 47, $558-561$.

26. Kresse, G.; Furthmüller, J., Efficient Iterative Schemes for Ab Initio Total-Energy Calculations Using a Plane-Wave Basis Set. Phys. Rev. B 1996, 54, 11169-11186.

27. Bertrand, G. H. V.; Polovitsyn, A.; Christodoulou, S.; Khan, A. H.; Moreels, I., Shape Control of Zincblende CdSe Nanoplatelets. Chem. Commun. 2016, 52, 11975-11978.

28. Lide, D. R., CRC Handbook of Chemistry and Physics. 84 ${ }^{\text {th }}$ ed.; CRC Press: 2003-2004.

29. Tessier, M. D.; Dupont, D.; De Nolf, K.; De Roo, J.; Hens, Z., Economic and Size-Tunable Synthesis of InP/ZnE (E = S, Se) Colloidal Quantum Dots. Chem. Mater. 2015, 27, 4893-4898.

30. Lauth, J.; Strupeit, T.; Kornowski, A.; Weller, H., A Transmetalation Route for Colloidal GaAs Nanocrystals and Additional III-V Semiconductor Materials. Chem. Mater. 2013, 25, 1377-1383.

31. Kim, M. R.; Miszta, K.; Povia, M.; Brescia, R.; Christodoulou, S.; Prato, M.; Marras, S.; Manna, L., Influence of Chloride Ions on the Synthesis of Colloidal Branched CdSe/CdS Nanocrystals by Seeded Growth. ACS Nano 2012, 6, 11088-11096.

32. Schliehe, C.; Juarez, B. H.; Pelletier, M.; Jander, S.; Greshnykh, D.; Nagel, M.; Meyer, A.; Foerster, S.; Kornowski, A.; Klinke, C.; Weller, H., Ultrathin PbS Sheets by Two-Dimensional Oriented Attachment. Science 2010, 329, 550-553.

33. Gerdes, F.; Navío, C.; Juárez, B. H.; Klinke, C., Size, Shape, and Phase Control in Ultrathin CdSe Nanosheets. Nano Lett. 2017, 17, 4165-4171.

34. Ghosh, S.; Manna, L., The Many "Facets" of Halide Ions in the Chemistry of Colloidal Inorganic Nanocrystals. Chem. Rev. 2018, 118, 7804-7864.

35. Reichardt, C., Solvents and Solvent Effects in Organic Chemistry. WILEY-VCH Verlag GmbH \& Co. KGaA: Weinheim, 2003; p 629. 


\section{Table of Content}

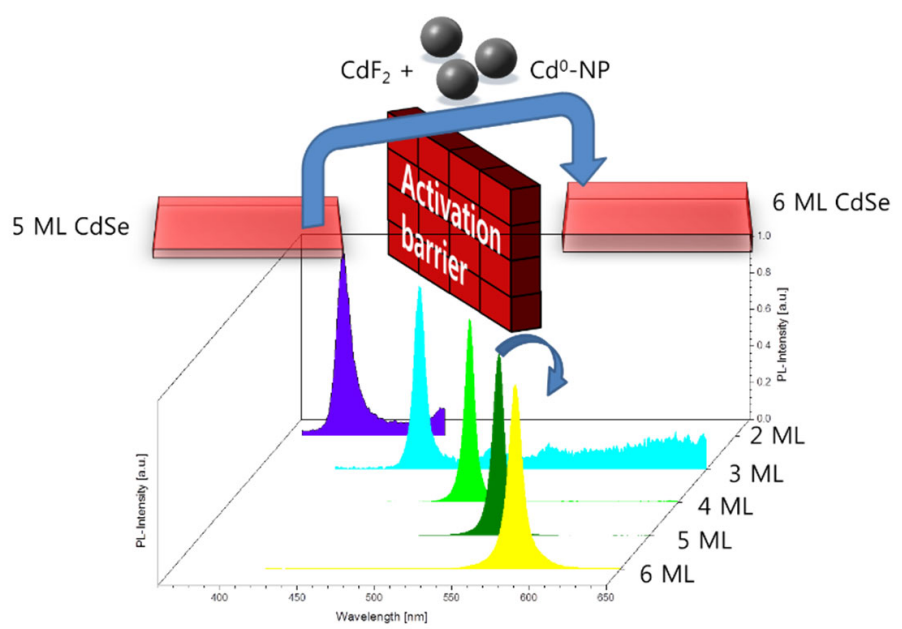

